Research Article

\title{
Effects of Crimp and Textile Architecture on the Stiffness and Strength of Composites with 3D Reinforcement
}

\author{
Fredrik Stig ${ }^{1,2}$ and Stefan Hallström (D) \\ ${ }^{1}$ KTH Royal Institute of Technology, Department of Aeronautical and Vehicle Engineering, SE 10044 Stockholm, Sweden \\ ${ }^{2}$ RISE SICOMP, SE 431 53, Mölndal, Sweden \\ Correspondence should be addressed to Stefan Hallström; stefanha@kth.se
}

Received 30 November 2018; Accepted 26 January 2019; Published 25 February 2019

Academic Editor: Lijing Wang

Copyright (C) 2019 Fredrik Stig and Stefan Hallström. This is an open access article distributed under the Creative Commons Attribution License, which permits unrestricted use, distribution, and reproduction in any medium, provided the original work is properly cited.

\begin{abstract}
The aim of this study is to experimentally determine how the weave architecture and yarn crimp affect the measured tensile stiffness and strength of composites containing 3D textile reinforcement. It is shown that both the stiffness and strength decrease nonlinearly with increasing $3 \mathrm{D}$ crimp. The ultimate strength of specimens containing nominally straight yarns and specimens containing crimped yarns can differ more than a factor of 3 , and the stress causing onset of damage can be affected even more. Adding nominally straight stuffer yarns into a 3D-woven reinforcement significantly increases the fibre volume fraction, the stiffness, and the strength of the composite. However, since the stuffer yarns are virtually straight and thus stiffer than the warp yarns, they attract the load and reach their strength at relatively lower strain than the warp yarns. The reinforcement architecture varies between the surfaces and the interior of the studied textiles, which has corresponding influence on the local stiffness. The onset of failure is predicted satisfactorily accurate with relatively simple estimations. The ultimate strength is a result of extensive damage progression and thus more dubious to predict.
\end{abstract}

\section{Introduction}

The current trend within aerospace and other transport industries is to increase the use of carbon fibre-reinforced composite materials to facilitate lower structural weight, better fuel economy, and lower emissions. The introduction of composite materials brings about not only several potential benefits but also a number of challenges. Composite materials are anisotropic and nonhomogeneous, and a common failure mode for two dimensionally (2D) laminated composites is delamination, associated with their relatively poor out-of-plane strength. Various three-dimensional (3D) textile reinforcements that partly suppress this problem have been developed, such as braiding, noobing $[1,2]$ (also referred to as 3D orthogonal weaves), 3D weaving [3], and different forms of 2D-woven 3D fabrics such as layer-tolayer and through-thickness angle interlock weaves. In this work, special attention is given to $3 \mathrm{D}$ weaving and noobing, according to the definitions set forth by Khokar [3].
The benefits with 3D-reinforced textile composites in comparison with their $2 \mathrm{D}$ counterparts are primarily improvements of out-of-plane properties, both in tension and in shear [4]. 3D weaving also enables net-shape manufacturing of complex 3D preforms directly from yarns, which could also reduce material waste substantially. Such preforms could be put in moulds for immediate impregnation into finished composite profiles, while using conventional $2 \mathrm{D}$ textiles would typically require stacking, preforming and bonding, and stapling or stitching before moulding.

There are however also drawbacks with the use of 3D textile reinforcements. The weaving process (for woven textiles) and the mere presence of through-thickness fibres (nonwovens) cause undulation of the in-plane fibres. This yarn crimp has a negative influence on both the stiffness and strength of the composite materials. Another drawback is the lack of knowledge and experience with these new materials and associated challenges to predict and express their material properties [5]. 
In this study, the stiffness and strength of 3D-reinforced composite materials with varying warp yarn crimp and different weave architectures are investigated experimentally and compared. The aims are as follows:

(i) To determine how (3D) yarn crimp affects the stiffness and the strength

(ii) To evaluate the difference in stiffness due to local architecture (since the local architecture in the interior and at the surfaces of the preforms differs)

(iii) To evaluate the stiffness and strength of composites reinforced with noobed fabrics (containing noninterlaced yarns in three perpendicular directions) and with $3 \mathrm{D}$ weaves containing stuffer yarns (noninterlaced, straight yarns in the warp direction)

The stiffness of 3D-reinforced composites has been fairly extensively studied both experimentally, e.g., [6-9], and through various models, e.g., [10-14]. The strength of composites reinforced with noobed and 2D-woven $3 \mathrm{D}$ fabrics has been studied in [7, 15-23], and composites containing $3 \mathrm{D}$-woven reinforcement were also studied in $[4,13]$.

\section{Experiments}

The specimens used for ultimate strength testing in this study were previously used for nondestructive stiffness measurements $[13,24]$. A plain 3D-woven reinforcement pattern was used, principally illustrated in where also representative volume elements (RVE) of the textile architecture are outlined.

The warp consisted of $8 \times 8$ Torayca T700 12k yarns and the weft of Toho Tenax HTA40 E13 6k yarns. For a more detailed description of the plain 3D weave and its internal geometry, the reader is referred to the study in [25]. All composite specimens were manufactured using vacuumassisted resin infusion in a rigid closed mould, using Dion 9500 vinyl ester.

Specimens with different warp crimp were produced by varying the weft density in three different ways in the $3 \mathrm{D}$ woven reinforcement. The intermediate weft density was used as reference for the entire test series and was thus labelled baseline (B). Reinforcement preforms with lower and higher crimp were labelled sparse (S) and dense (D), respectively. The global fibre volume fraction $v_{\mathrm{f}}$ was kept constant for the different composite specimens, regardless of the crimp, by adjusting the cross section area of the mould cavity to the preform fibre content. Here crimp is defined as the physical length of the yarn along its path, $L_{y}$, over the corresponding wavelength $\lambda$, as follows:

$$
\operatorname{crimp}=\frac{L_{\mathrm{y}}}{\lambda} \text {. }
$$

To investigate if any cross section size effects occurred, two thinner versions of the baseline weave type were also produced, containing only $6 \times 6$ and $4 \times 4$ warp yarns. These specimens were correspondingly labelled $6 \times 6 \mathrm{~B}$ and $4 \times 4 \mathrm{~B}$, respectively.

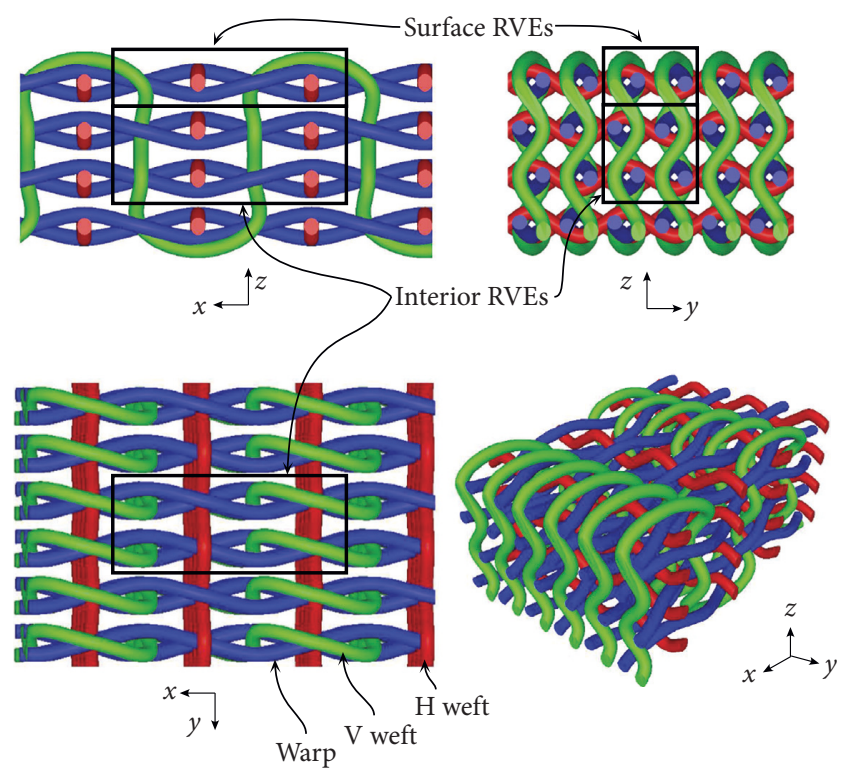

FIgUre 1: A plain 3D weave, comprising warp, horizontal $\mathrm{H}$, weft and vertical $\mathrm{V}$ weft, depicted along its three principal axes and in an isometric view. Surface and interior RVEs of the weave architecture are also outlined.

In addition, specimens reinforced with plain 3D weaves with incorporated stuffer yarns and with noobed 3D reinforcement were manufactured for comparison. The textile preforms were produced with the same loom settings and thus the same weft density, as the baseline configuration.

The 3D-woven preforms containing stuffer yarns are labelled BS. Nominally straight Torayca T700 12k stuffer yarns were inserted in the pockets between the warp yarns in the plain $3 \mathrm{D}$ weave, visible in the upper right illustration in Figure 1. The yarn content was significantly increased by the presence of the stuffer yarns, and hence $v_{\mathrm{f}}$ was considerably higher for the BS than for the B specimens.

The specimens with noobed $3 \mathrm{D}$ reinforcement are labelled BN. The noobed textile geometry also contains yarns in three mutually perpendicular directions, but the yarns are not interlaced within the interior representative volume element (RVE) (Figure 2). As a consequence, all yarns in the principal $(x)$ direction are nominally straight.

For all specimens, regardless of type, the textile architecture at the surfaces differs from the interior RVEs, as seen in Figures 1 and 2.

With the exception of reinforcement type $\mathrm{D}$, the yarn packing and thus the fibre volume fraction were slightly higher in the interior RVEs than at the surfaces, for all specimens containing $3 \mathrm{D}$ textile reinforcements. The difference was most pronounced for the noobed specimens, associated with the compact noobed fabric architecture. On the contrary, all textile reinforcements had a larger share of yarns oriented in the principal $(x)$ direction at the surfaces since the wefts are traversing a short distance between every pick (Figures 1 and 2).

All specimens had square cross sections, which is not ideal for strength testing since the available area for griping is small in comparison to the cross section area and, as a 


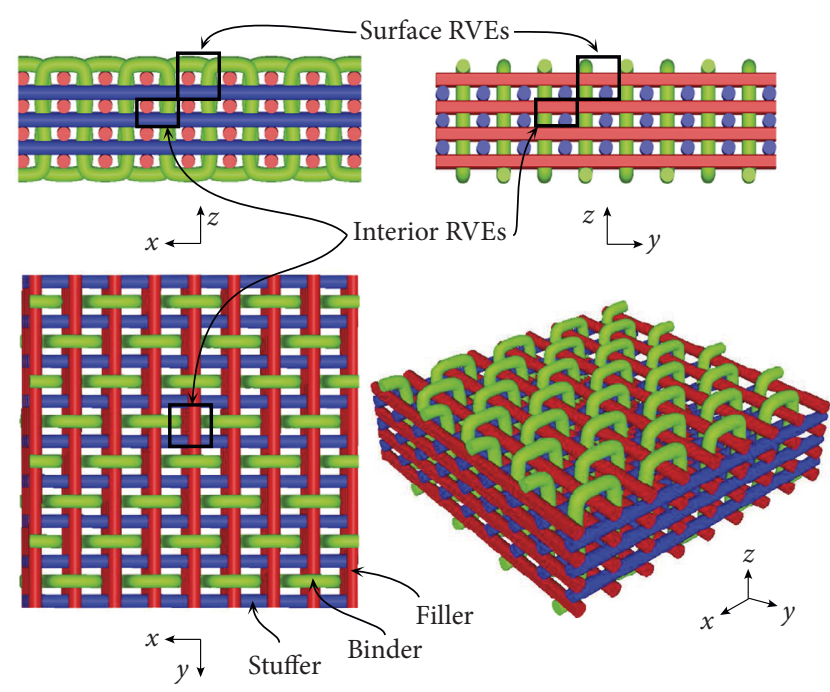

Figure 2: A noobed fabric seen from its three principal planes and in an isometric view. Surface and interior RVEs are also outlined.

consequence, the load required to break the material. All specimens were therefore milled down to a dog-bone shape, as illustrated in Figure 3, in order to reduce the tested cross section area and enable strength testing. The intention was to mill off only the surface unit cells in order to obtain stiffness and strength data for the interior RVEs.

$\mathrm{X}$-ray computed tomography (CT) images of the specimen cross sections were used to estimate the average thickness of the surface (RVE) layers. Based on such estimates, $1.3 \mathrm{~mm}$ was milled off from each side of the specimens. As can be seen in Figure 4, the reinforcement was not perfectly centered and evenly distributed over the specimen cross sections, which made the elimination of the surface RVEs somewhat approximate. The letter $\mathrm{M}$ in the type name indicates that specimens were milled, in the sense that a pristine baseline $8 \times 8$ warp specimen $(8 \times 8 \mathrm{~B})$ would be denoted $6 \times 6 \mathrm{BM}$ after milling.

Analysis of CT and microscopy images also revealed that the warp layers in the 3D-woven reinforcement were not distinctly separated into plies that easily could be removed, but rather mixed. The packing of the textile reinforcement is in fact higher than that outlined in Figure 1, and a more representative illustration is provided in Figure 5.

The milling operation might thus not only remove the outermost layer of warp yarns but also damage underlaying yarns in the second warp layer. Such damage is likely to have a greater impact on the strength than on the stiffness since it does not affect the fibre volume fraction much, but it chops up the warp yarns near the surfaces.

The Young modulus prior to milling (pristine specimens) was first measured. Then, the Young modulus of the milled samples (intended to contain only interior RVEs) was also measured. Using these results, the stiffness of the surface unit cells $\left(E_{\text {surf }}\right)$ could be evaluated as

$$
E_{\text {surf }}=\frac{E-v_{\text {int }} E_{\text {int }}}{\left(1-v_{\text {int }}\right)}
$$

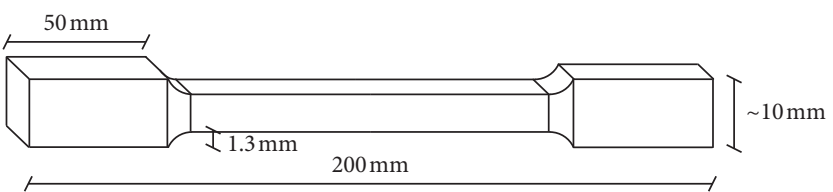

Figure 3: Principal geometry of the milled dog-bone test specimens.

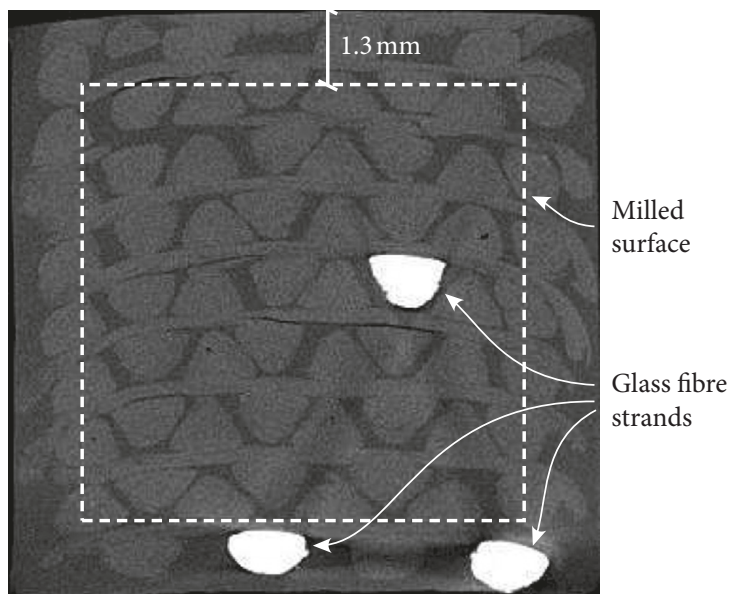

Figure 4: A CT scan image of an $8 \times 8 \mathrm{~B}$ specimen cross section. The dashed lines indicate the reduction of the cross-sectional area from milling. (Some glass fibre strands were integrated in the CT specimens, for other visualisation purposes. No glass was present in the tested specimens).

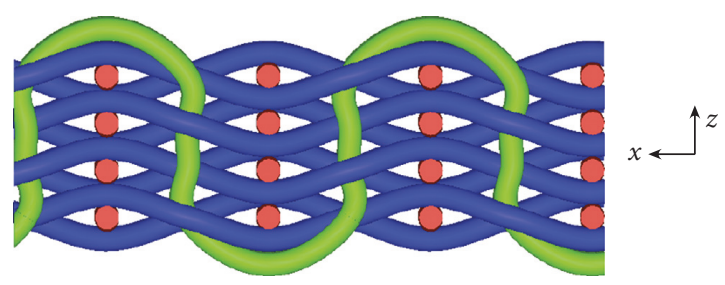

FIgURE 5: True packing of the 3D-woven reinforcement, illustrating the presence of partial overlap between warp layers.

where $E$ is the Young modulus of the pristine specimens, $v_{\text {int }}$ is the volume fraction of interior RVEs, and $E_{\text {int }}$ is the modulus of the milled specimens.

General information about the weave types and the number of specimens of each category is presented in Table 1. Note that stiffness measurements were performed both prior to and after milling, while only milled specimens were used for strength testing. The fibre volume fraction of all tested specimens was either measured or estimated from the fibre content and the dimensions of the RVEs.

Various gripping and load introduction arrangements were designed and evaluated in a previous study [26], such as epoxy cast edges and different combinations of pins, grooves, teeth, tabs, and wedges. In spite of considerable efforts, plain tensile testing with standard machine grippings turned out to work best. 
TABle 1: Test specimen data, including warp yarn crimps, fibre volume fractions, and mean warp offset angles.

\begin{tabular}{lccccc}
\hline \multirow{2}{*}{$\begin{array}{l}\text { Textile type } \\
\text { Name }\end{array}$} & \multicolumn{3}{c}{ Plain 3D woven } & Noobed & $\begin{array}{c}\text { 3D woven } \\
\text { with stuffer }\end{array}$ \\
\hline Mean warp crimp & 1.012 & 1.025 & 1.055 & 1 & $1.031^{*}$ \\
$v_{\mathrm{f}}$ & 0.43 & 0.42 & 0.42 & 0.44 & 0.57 \\
$v_{\mathrm{f}}$ (interior RVE) & 0.45 & 0.45 & 0.42 & 0.50 & 0.59 \\
$\theta(\mathrm{deg})^{* *}$ & 8.8 & 12.8 & 18.6 & $\approx 0$ & - \\
$8 \times 8$ & 2 & 4 & 3 & 2 & 2 \\
$6 \times 6 \mathrm{M}$ & $2(1)$ & $4(3)$ & $3(3)$ & $2(1)$ & $2(1)$ \\
$6 \times 6$ & - & 4 & - & 2 & - \\
$4 \times 4 \mathrm{M}$ & - & $4(2)$ & - & $2(2)$ & - \\
$4 \times 4$ & - & 2 & - & - & - \\
\hline
\end{tabular}

The number of test specimens for each configuration is presented in the lower half of the table, where numbers within brackets indicate the number of specimens that eventually were tested to tensile failure. ${ }^{*}$ BS also contains stuffer yarns with crimp $\approx 1 .{ }^{* *}$ Average warp angle with respect to the $x$ direction.

All tests were conducted at room temperature and at a constant displacement rate of $2 \mathrm{~mm} / \mathrm{min}$ in a $100 \mathrm{kN}$ Instron universal test machine equipped with a $100 \mathrm{kN}$ load cell. Strains were measured using both a $50 \mathrm{~mm}$ strain gauge and a digital image correlation (DIC) system. With the exception for the geometry of the test specimens, the procedure followed the ASTM D3039 [27] test standard.

\section{Strength Estimates}

The stress vs. strain response of composites containing woven reinforcement often shows a pronounced nonlinear behavior prior to ultimate stress. The phenomenon was denoted a plastic tow straightening by Cox et al. [15] and could be regarded as an onset of failure, characterised by plastic softening of the material. In this work, an impregnated tow is referred to as a strand, and the phenomenon believed to be associated with initiation of matrix cracks within the strands caused by shear stresses [15]. The type of softening was observed previously in [4] for composites containing 2D and 3D weaves, and in works by $[7,15,16]$ similar testing was performed on composites containing noobed and 3D interlock weaves.

Cox et al. [15] proposed an expression for estimating the tensile stress level at which plastic tow straightening initiates, assuming small yarn undulations. In the following, similar relations are derived but without any restrictions on the crimp angles. It is assumed that the tensile load is carried entirely by the strands in the warp direction. Letting $x$ represent the nominal warp direction and assuming a tensile force $F_{x}$ acting in that direction, the following relation is obtained:

$$
F_{x}=\sigma_{x}^{a} A_{\text {tot }} \approx \sigma_{x}^{s} A_{\text {warp }}^{s},
$$

where $\sigma_{x}^{a}$ is the average stress and $\sigma_{x}^{s}$ is the stress inside the warp strands. The total cross-sectional area of the warp strands, projected on the $y z$ plane, is denoted $A_{\text {warp }}^{s}$ while the corresponding projection of the total cross-sectional area of the RVE is denoted $A_{\text {tot }}$. Cox et al. [15] suggested that the onset of plastic straightening initiates when the warp strands fail in shear. The shear stress in the strands, induced by the tensile stress $\sigma_{x}^{s}$, is given in a local 1-2 coordinate system by

$$
\left|\tau_{12}^{s}\right|=\sin \theta \cos \theta \sigma_{x}^{s},
$$

where the 1 axis coincides with the local warp yarn orientation and $\theta$ is the mean warp offset angle between the local 1 and the global $x$ axis. The onset stress for plastic tow straightening is then obtained by combining eqs. (3) and (4) and replacing the shear stress with the shear strength of the strand $\widehat{\tau}_{12}^{s}$ :

$$
\sigma_{x}^{\text {onset }} \approx \frac{\widehat{\tau}_{12}^{s}}{\cos \theta \sin \theta} \frac{A_{\text {warp }}^{s}}{A_{\text {tot }}} .
$$

Shear strength values $\widehat{\tau}_{12}^{s}$ ranging between $62 \mathrm{MPa}$ and $80 \mathrm{MPa}$ have been reported for carbon fibre-reinforced plastic (CFRP) laminates [15, 28, 29]. Here, a mean value $(72 \mathrm{MPa})$ was assumed based on the fibre volume fraction within the strands $v_{f}^{s}$ being similar to the reported fibre volume fractions in $[15,28,29]$. The strand cross-sectional area $A_{\text {warp }}^{s}$ was obtained from [13]. The remaining input is listed in Table 1, where the mean angle $\theta$ with respect to the $x$ direction is estimated assuming a helical warp strand trajectory [13].

\section{Results and Discussion}

4.1. Stiffness Measurements. The stiffness was measured for all specimens, first before milling, with specimens containing interior and surface RVEs, and then after the surface layers had been milled off, leaving only interior RVEs in the specimens (only interior RVEs). The results from the model of the warp yarn crimp are presented in Figure 6, where the crimp values originate from [13] for all specimens containing plain 3D weave. The noobed reinforcement is considered straight $(\mathrm{crimp}=1)$, although there was likely minor yarn undulation in the specimens [30].

The stiffness of the surface RVEs was then estimated for each reinforcement type using eq. (2) and the two measured stiffnesses for pristine and milled specimens. The fraction of interior RVEs $v_{\text {int }}$ was estimated as the ratio between the interior and the total number of warp yarns, i.e., 36/64 for $8 \times 8$ specimens and $16 / 36$ for $6 \times 6$ specimens. The results are presented in Table 2 .

The stiffness of the interior RVE does not vary with the cross section area of the tested specimens for cases where they can be compared, i.e., B and BN. The difference in stiffness between the surface and interior unit cells varies with the weft density. The surface stiffness appears to be higher for the $S$ specimens and lower for the D specimens and somewhere in between for the baseline case (B). The surface stiffness variation thus follows the interior stiffness variation, but the relative change is lower, making the surface stiffer than the interior for the $\mathrm{D}$ specimens and vice versa for the $S$ specimens, whereas the surface and interior stiffness is relatively similar for the $\mathrm{B}$ case. For the specimens containing noobed reinforcement (BN), neither the interior nor the surface stiffness varies with the cross-sectional area, 


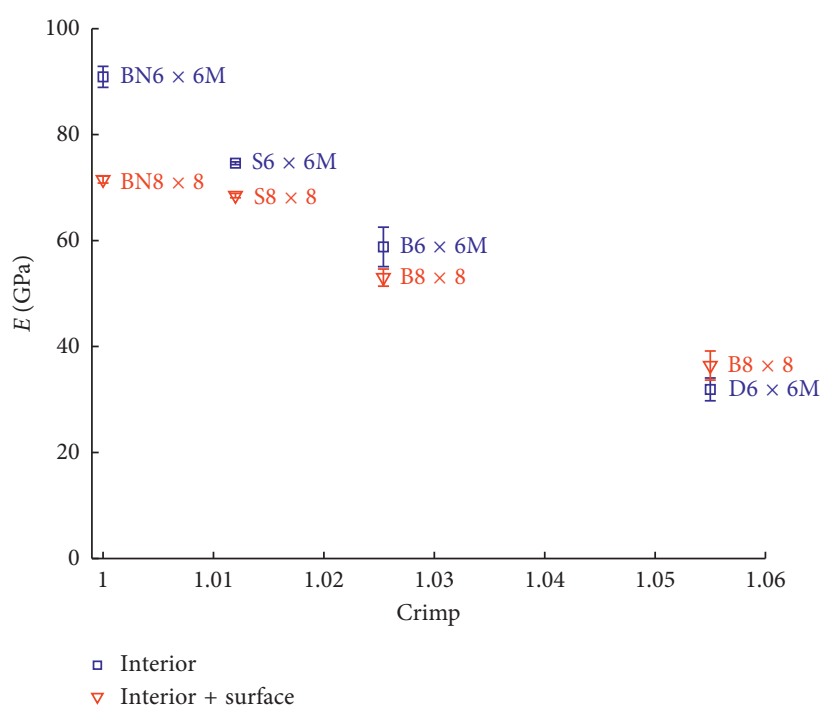

FIGURE 6: The Young modulus of composite specimens containing 3D-woven and noobed reinforcement. Results from both pristine (I + S) and milled (I) specimens are presented.

TABle 2: The Young moduli (GPa), measured and estimated from equation (2).

\begin{tabular}{|c|c|c|c|c|c|c|}
\hline \multicolumn{2}{|l|}{ Textile type } & \multicolumn{3}{|c|}{ Plain 3D woven } & \multirow{2}{*}{$\begin{array}{c}\text { Noobed } \\
\text { BN }\end{array}$} & \multirow{2}{*}{$\begin{array}{c}\text { 3D woven with stuffer } \\
\text { BS }\end{array}$} \\
\hline Name & Warp & $S$ & B & $\mathrm{D}$ & & \\
\hline \multirow{3}{*}{ Measured, pristine $(S+I)$} & $8 \times 8$ & $68.5 \pm 0.4$ & $53.0 \pm 1.6$ & $36.4 \pm 2.8$ & $71.5 \pm 0.7$ & $79.2 \pm 6.7$ \\
\hline & $6 \times 6$ & - & $57.5 \pm 1.3$ & - & $67.8 \pm 1.5$ & - \\
\hline & $4 \times 4$ & - & $57.7 \pm 0.1$ & - & - & - \\
\hline \multirow{2}{*}{ Measured, milled (I) } & $6 \times 6 \mathrm{M}$ & $74.6 \pm 0.3$ & $58.8 \pm 5.1$ & $31.9 \pm 2.2$ & $90.5 \pm 2.4$ & 96.4 \\
\hline & $4 \times 4 \mathrm{M}$ & - & $58.9 \pm 2.5$ & - & $91.2 \pm 2.4$ & - \\
\hline \multirow{2}{*}{ Estimated (S) } & $8 \times 8$ & 60.7 & 45.6 & 42.2 & 47.1 & 57.1 \\
\hline & $6 \times 6$ & - & 56.4 & - & 49.1 & - \\
\hline
\end{tabular}

and due to their lower crimp, they are consistently stiffer than the specimens containing woven reinforcement. The interior stiffness is higher, and the surface stiffness appears to be similar to that of corresponding specimens with woven reinforcement. The specimens containing 3D-woven reinforcement and stuffer yarns (BS) show the highest interior stiffness, which seems sensible since these specimens are also extreme in terms of yarn content in the warp $(x)$ direction.

No obvious reason for the somewhat low stiffness results achieved from the tests of the $\mathrm{B} 8 \times 8$ specimens was observed. It is possible that the $\mathrm{B} 6 \times 6 \mathrm{M}$ results are misleadingly high, given the relatively high standard deviation. A higher stiffness for $\mathrm{B} 8 \times 8$ or a lower stiffness for $\mathrm{B} 6 \times 6 \mathrm{M}$ would result in a higher estimated surface stiffness for $\mathrm{B} 8 \times 8$, which would be more consistent with the results from the other configurations.

Denser weave packing actually causes more void in the textile architecture and thus more matrix material and a lower fibre volume fraction in the interior RVEs of the impregnated composite specimens. Since the overall fibre volume fraction is kept similar for the $\mathrm{S}, \mathrm{B}$, and $\mathrm{D}$ specimens, the fibre volume fraction in the surface layers increases with the weave density. That is not accounted for in the estimates of the stiffness of the surface layers, which might also contribute to the minor inconsistencies in the stiffness results.

4.2. Strength Measurements. The plain 3D weave specimens all exhibit nonlinear stress vs. strain curves typical for plastic tow straightening. Estimates of when the onset of plastic tow straightening occurs were made using equation (5). A stressstrain curve for a milled baseline specimen (BM) is presented in Figure 7, together with the corresponding estimate. A straight line indicating the stiffness is also included in the graph. All results are summarised in Table 3.

As can be seen, there is good agreement for the SM and the $\mathrm{BM}$ reinforcements, while for the most heavily crimped sample (DM), there is a significant difference. Both the experimental results in Table 3 and eq. (5) indicate that the increasing crimp (and correspondingly increasing warp yarn offset angle $\theta$ ) decreases the stress at which the onset of plastic tow straightening occurs.

For the noobed BNM specimens with nominally straight reinforcement, the stress-strain curves are essentially linear until failure, as illustrated in Figure 8.

The BS reinforcement contains both warp and stuffers in the direction $(x)$ of the applied load. The warp has similar 


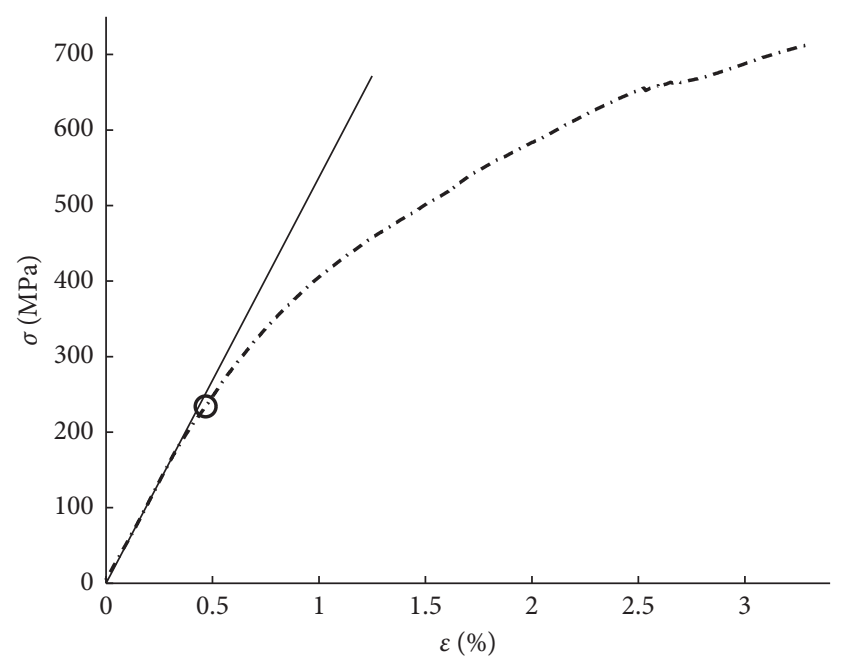

FIgURE 7: Experimental stress-strain curve of a milled baseline specimen (BM) with the predicted onset of plastic tow straightening (circle) and the initial stiffness (thin straight line) indicated.

TABLE 3: Experimentally determined and estimated stress levels where the onset of plastic tow straightening occurs.

\begin{tabular}{lccc}
\hline Name & SM & BM & DM \\
\hline Estimated value (MPa) & 347 & 228 & 144 \\
Experimental value (MPa) & $\approx 370$ & $200-260$ & $\approx 100$ \\
\hline
\end{tabular}

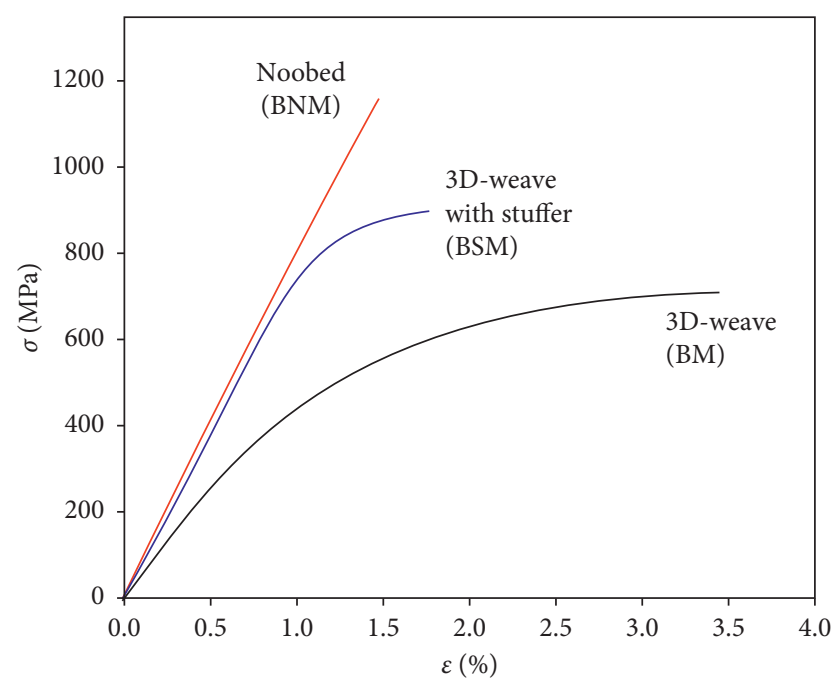

FIGURE 8: The principal stress vs. strain response of specimens with noobed (BNM) reinforcement and 3D-woven reinforcement with stuffer yarns (BSM). The principal response of a specimen reinforced with a plain 3D weave (BM) is added for comparison. The curves are valid for interior RVEs since all specimens were milled.

crimp as the warp in the type B reinforcement, while the stuffers running in parallel are virtually straight. The stuffers are therefore in effect stiffer and tend to carry a considerably larger share of the applied load than the warp. They are also expected to fail at lower applied tensile strain than the warp since they are entirely aligned with the loading direction. As a consequence, the BS specimens are expected to show a sequential tensile failure where the load is initially carried predominantly by the stuffers and then gradually transferred to the warp, when the stuffers start to fail. This transition is also supported by experimental results as seen in Figure 8 . The plastic tow straightening effect appears to be suppressed, which might be explained by the stuffers carrying the main part of the applied load. Eventually, the stress-strain curve shows a clear shift at about $70 \%$ of the ultimate stress, when cracking sounds were also recorded during testing. It is believed that the shift and the cracking sounds were associated with tensile failure of the stuffer yarns.

The ultimate strength for the only BSM specimen that was tested to tensile failure (Figure 8) was $1032 \mathrm{MPa}$, which is $53 \%$ higher than the average strength for corresponding specimens without stuffer yarns (BM). The relative difference in onset of damage was even greater and happened at approximately 3 times higher stress. This seems reasonable considering that the BS reinforcement contains additional yarns in the warp direction and also has a higher fibre volume fraction compared to the $\mathrm{B}$ reinforcement. The ultimate strength for the BNM specimens was however yet higher, $1371 \mathrm{MPa}$ in average, in spite of lower fibre content in the warp direction and lower fibre volume fraction than the BSM specimens. This is also considered reasonable since all of the noobed reinforcement is straight and aligned, which generates a more even stress distribution in the material.

The ultimate strength as function of crimp for specimen types SM, BM, DM, and BNM is presented in Figure 9, where yarn crimp $=1$ is assumed for the BNM specimens. No significant difference in strength was observed for the $6 \times 6 \mathrm{M}$ and $4 \times 4 \mathrm{M}$ specimens for neither of the $\mathrm{B}$ and $\mathrm{BN}$ reinforcements, and all results are thus presented together for the two reinforcement types, respectively. The overall trend is that the strength decreases with increasing crimp, in a similar fashion as the stiffness, previously presented in Figure 6.

The strain at ultimate failure of the specimens containing $3 \mathrm{D}$-woven reinforcement is high, which is in agreement with test results for other composites containing 3D reinforcement found in the literature $[15,20]$. These strain levels should however not be quoted when referring to the strength of the materials since damage initiates at much lower strains. The BNM specimens show more moderate strains to failure due to their noobed reinforcement being straight and aligned with the tensile load.

Due to the progressive damage, the ultimate strength of the specimens with woven reinforcement is dubious to predict using established failure criteria. It was noted in this work that failure according to, for example, the Tsai-Hill criterion provides almost identical predictions as the expression for plastic tow straightening in equation (5), which are far below measured values of the ultimate strength. This is reasonable since the shear strength within the strands dominate the Tsai-Hill criterion for the present load case. If, on the contrary, it is assumed that the ultimate strength is solely dependent on the longitudinal fibre strength, then the overall strength is significantly overestimated. Cox et al. [16] also stressed the difficulty to predict the ultimate strength of textile composites, 


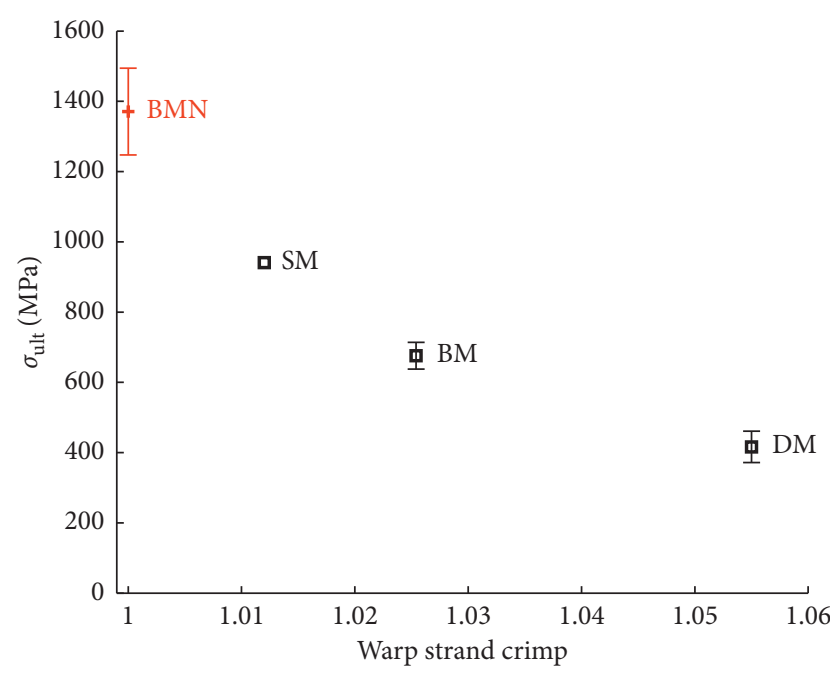

FIGURE 9: Ultimate strength as function of the warp yarn crimp for the specimens containing 3D-woven and noobed reinforcement.

attributing the discrepancies between predictions and experimental data to yarn damage from weaving, and local misalignment of the strands that could be caused both during weaving and impregnation. Regardless of yarn damage and misalignment, more accurate predictions of the ultimate strength likely require elaborate models of the progressive damage observed in this study.

\section{Conclusions}

Both the stiffness and the ultimate strength of composite materials containing $3 \mathrm{D}$ textile reinforcement decrease substantially with increasing yarn crimp. In particular, three-dimensional warp yarn crimp has a great influence on the tensile strength of composites containing 3D-woven reinforcement. The ultimate strength of specimens containing noobed reinforcement (BNM) and the most heavily crimped 3D-woven reinforcement (DM) differed more than a factor of 3, and the onset of damage differed even more.

As a consequence, there is no reason to use more through-thickness reinforcement in 3D textiles than necessary (from requirements on out-of-plane stiffness and strength), since the higher crimp associated with a denser weave pattern is so detrimental to the in-plane properties. One should also consider use of more favourable weave patterns to limit the overall crimp. There is thus no point in using 3D reinforcement where it is not needed to provide interlacement and avoid delaminations. The 3D-weaving technique permits a mix of $2 \mathrm{D}$ and $3 \mathrm{D}$ reinforcement within the same preform, so areas that are primarily subjected to in-plane loading could contain $2 \mathrm{D}$ reinforcement only.

For the reinforcement studied in this work, the architecture varied between the surfaces and the interior of the textile preforms, which was shown to generate corresponding stiffness variations in the composite materials.

The strain at ultimate load of composites containing plain 3D-woven reinforcement is high but should be considered with caution since shear cracks in the warp strands start to initiate at much lower strain levels, and from then on, the stress-strain response is clearly nonlinear and associated with damage growth.

Including nominally straight stuffer yarns in the $3 \mathrm{D}$ weave increases not only the overall fibre content but also the fibre volume fraction, due to more efficient yarn packing in the textiles. The presence of stuffer yarns appears to suppress the initiation of shear cracks in the warp. Specimens with 3D-woven reinforcement, including stuffer yarns, started to fail at considerably higher stress and strain levels than corresponding specimens without stuffers. When they eventually started to fail, they lost their linear stress-strain response, in a similar fashion than their counterparts without stuffers.

\section{Data Availability}

No data were used to support this study.

\section{Disclosure}

The work was performed as a part of F. Stig's doctoral thesis entitled 3D-Woven Reinforcement in Composites [25].

\section{Conflicts of Interest}

The authors declare that they have no conflicts of interest.

\section{Acknowledgments}

This work was financially supported by the European Commission through the EU FP7 contract no. ACPO-GA2010-266026, Cost Effective Reinforcement of Fastener Areas in Composites (CERFAC). Biteam AB is acknowledged for supplying the $3 \mathrm{D}$-woven preforms.

\section{References}

[1] M. H. Mohamed, A. E. Bogdanovich, L. C. Dickinson, J. N. Singletary, and R. Bradley Lienhart, "A new generation of 3D woven fabric preform and composite," SAMPE Journal, vol. 37, no. 3, pp. 8-17, 2001.

[2] N. Khokar, "3D-weaving: theory and practice," Journal of the Textile Institute, vol. 92, no. 2, pp. 193-207, 2001.

[3] N. Khokar, 3D-Weaving and noobing: characterization of interlaced and non-interlaced 3D fabric forming principles, $\mathrm{PhD}$ thesis, Chalmers University of Technology, Gothenburg, Sweden, 1997.

[4] F. Stig and S. Hallström, "Assessment of the mechanical properties of a new 3D woven fibre composite material," Composites Science and Technology, vol. 69, no. 11-12, pp. 1686-1692, 2009.

[5] C. McHugh: Taking textiles to the next dimension, Aerospace Manufacturing, July 2011.

[6] D. S. Ivanov, S. V. Lomov, A. E. Bogdanovich, M. Karahan, and I. Verpoest, "A comparative study of tensile properties of non-crimp 3D orthogonal weave and multi-layer plain weave e-glass composites. part 2: comprehensive experimental results," Composites Part A: Applied Science and Manufacturing, vol. 40, no. 8, pp. 1144-1157, 2009. 
[7] P. J. Callus, A. P. Mouritz, M. K. Bannister, and K. H. Leong, "Tensile properties and failure mechanisms of 3D woven GRP composites," Composites Part A: Applied Science and Manufacturing, vol. 30, no. 11, pp. 1277-1287, 1999.

[8] B. N. Cox and M. S. Dadkhah, "The macroscopic elasticity of 3D woven composites," Journal of Composite Materials, vol. 29, no. 6, pp. 785-819, 2016.

[9] A. P. Mouritz and B. N. Cox, "A mechanistic interpretation of the comparative in-plane mechanical properties of $3 \mathrm{~d}$ woven, stitched and pinned composites," Composites Part A: Applied Science and Manufacturing, vol. 41, no. 6, pp. 709-728, 2010.

[10] I. Verpoest and S. Lomov, "Virtual textile composites software: integration with micro-mechanical, permeability and structural analysis," Composites Science and Technology, vol. 65, no. 15-16, pp. 2563-2574, 2005.

[11] J. Xu, B. N. Cox, M. A. McGlockton, and W. C. Carter, "A binary model of textile composites-II. The elastic regime," Acta Metallurgica et Materialia, vol. 43, no. 9, pp. 3511-3524, 1995.

[12] Z. Wu, "Three-dimensional exact modeling of geometric and mechanical properties of woven composites," Acta Mechanica Solida Sinica, vol. 22, no. 5, pp. 479-486, 2009.

[13] F. Stig and S. Hallström, "Influence of crimp on 3D-woven fibre reinforced composites," Composite Structures, vol. 95, pp. 114-122, 2013.

[14] S. Z. Sheng and S. van Hoa, "Modeling of 3D angle interlock woven fabric composites," Journal of Thermoplastic Composite Materials, vol. 16, no. 1, pp. 45-58, 2016.

[15] B. N. Cox, M. S. Dadkhah, and W. L. Morris, "On the tensile failure of 3D woven composites," Composites Part A: Applied Science and Manufacturing, vol. 27, no. 6, pp. 447-458, 1996.

[16] B. N. Cox, M. S. Dadkhah, W. L. Morris, and J. G. Flintoff, "Failure mechanisms of 3D woven composites in tension, compression, and bending," Acta Metallurgica et Materialia, vol. 42, no. 12, pp. 3967-3984, 1994.

[17] A. E. Bogdanovich, "Multi-scale modeling, stress and failure analyses of 3D woven composites," Journal of Materials Science, vol. 41, no. 20, pp. 6547-6590, 2006.

[18] J. Brandt, K. Drechsler, and F.-J. Arendts, "Mechanical performance of composites based on various three-dimensional woven-fibre preforms," Composites Science and Technology, vol. 56, no. 3, pp. 381-386, 1996.

[19] M. P. Rao, B. V. Sankar, and G. Subhash, "Effect of Z-yarns on the stiffness and strength of three-dimensional woven composites," Composites Part B: Engineering, vol. 40, no. 6, pp. 540-551, 2009.

[20] S. V. Lomov, A. E. Bogdanovich, D. S. Ivanov, D. Mungalov, M. Karahan, and I. Verpoest, "A comparative study of tensile properties of non-crimp 3D orthogonal weave and multilayer plain weave e-glass composites. part 1: materials, methods and principal results," Composites Part A: Applied Science and Manufacturing, vol. 40, no. 8, pp. 1134-1143, 2009.

[21] P. Tan, L. Tong, and G. P. Steven, "Behavior of 3D orthogonal woven CFRP composites. Part II. FEA and analytical modeling approaches," Composites Part A: Applied Science and Manufacturing, vol. 31, no. 3, pp. 273-281, 2000.

[22] L. Tong, P. Tan, and G. P. Steven, "Effect of yarn waviness on strength of 3D orthogonal woven CFRP composite materials," Journal of Reinforced Plastics and Composites, vol. 21, no. 2, pp. 153-173, 2002.

[23] S. Dai, P. R. Cunningham, S. Marshall, and C. Silva, "Influence of fibre architecture on the tensile, compressive and flexural behaviour of 3D woven composites," Composites Part A: Applied Science and Manufacturing, vol. 69, pp. 195-207, 2015.

[24] F. Stig and S. Hallström, "A modelling framework for composites containing 3D reinforcement," Composite Structures, vol. 94, no. 9, pp. 2895-2901, 2012.

[25] F. Stig, 3D-woven reinforcement in composites, $\mathrm{PhD}$ thesis, Royal Institute of Technology (KTH), Stockholm, Sweden, 2012.

[26] M. González Eizaguirre, "Tensile testing of 3D reinforced composites," Master's thesis, Royal Institute of Technology (KTH), Stockholm, Sweden, 2011.

[27] ASTM D 3039-00, Standard Test Method for Tensile Properties of Polymer Matrix Composite Materials, American Society for Testing and Materials, West Conshohocken, PA, USA, 2000.

[28] C. C. Chamis, "Simplified composite micromechanics equations for strength, fracture toughness and enviromental effects," SAMPE Quarterly, vol. 15, pp. 41-55, 1984.

[29] D. Zenkert and M. Battley, Foundations of Fibre Composites, KTH Aeronautical and Vehicle Engineering, Stockholm, Sweden, 1996.

[30] M. Karahan, S. V. Lomov, A. E. Bogdanovich, D. Mungalov, and I. Verpoest, "Internal geometry evaluation of non-crimp 3D orthogonal woven carbon fabric composite," Composites Part A: Applied Science and Manufacturing, vol. 41, no. 9, pp. 1301-1311, 2010. 


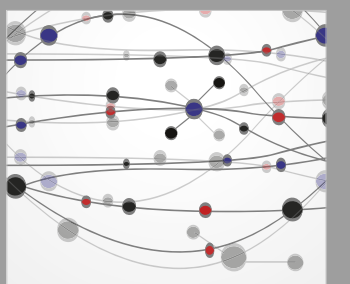

The Scientific World Journal
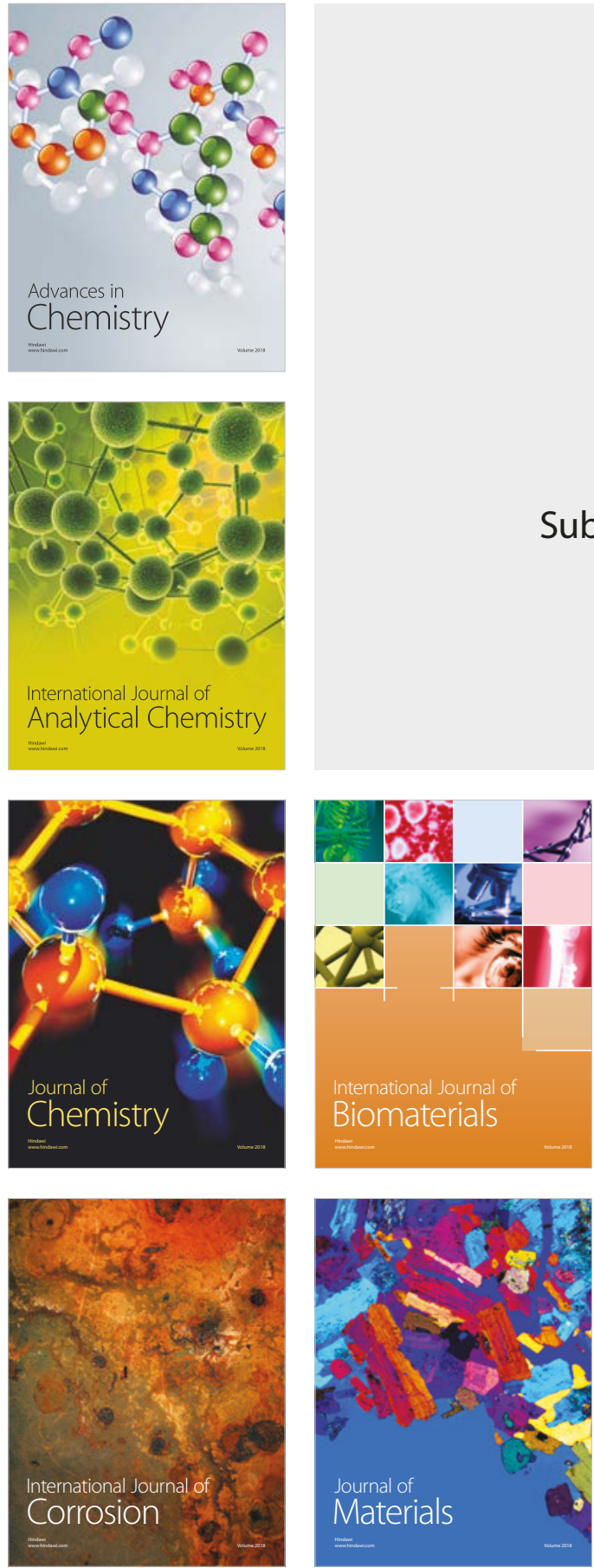

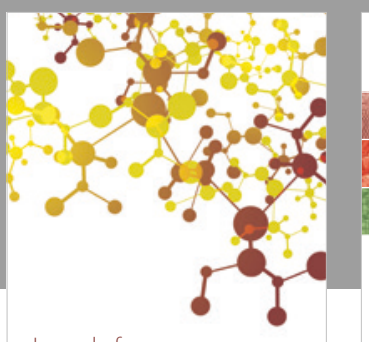

Journal of

Applied Chemistry
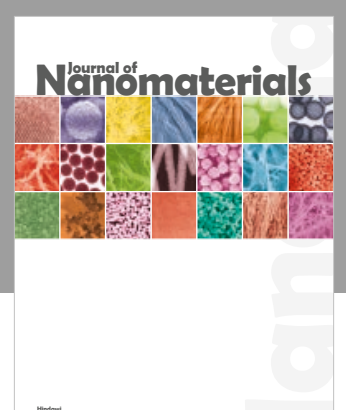

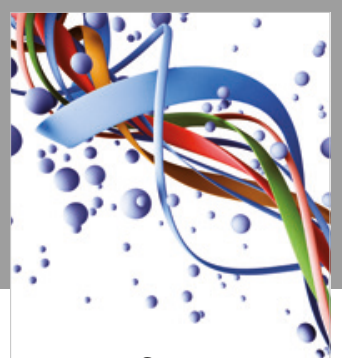

Scientifica

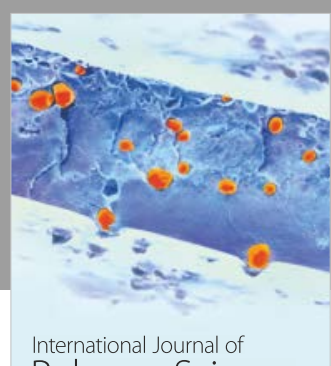

Polymer Science

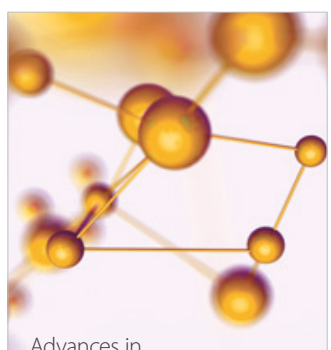

Physical Chemistry
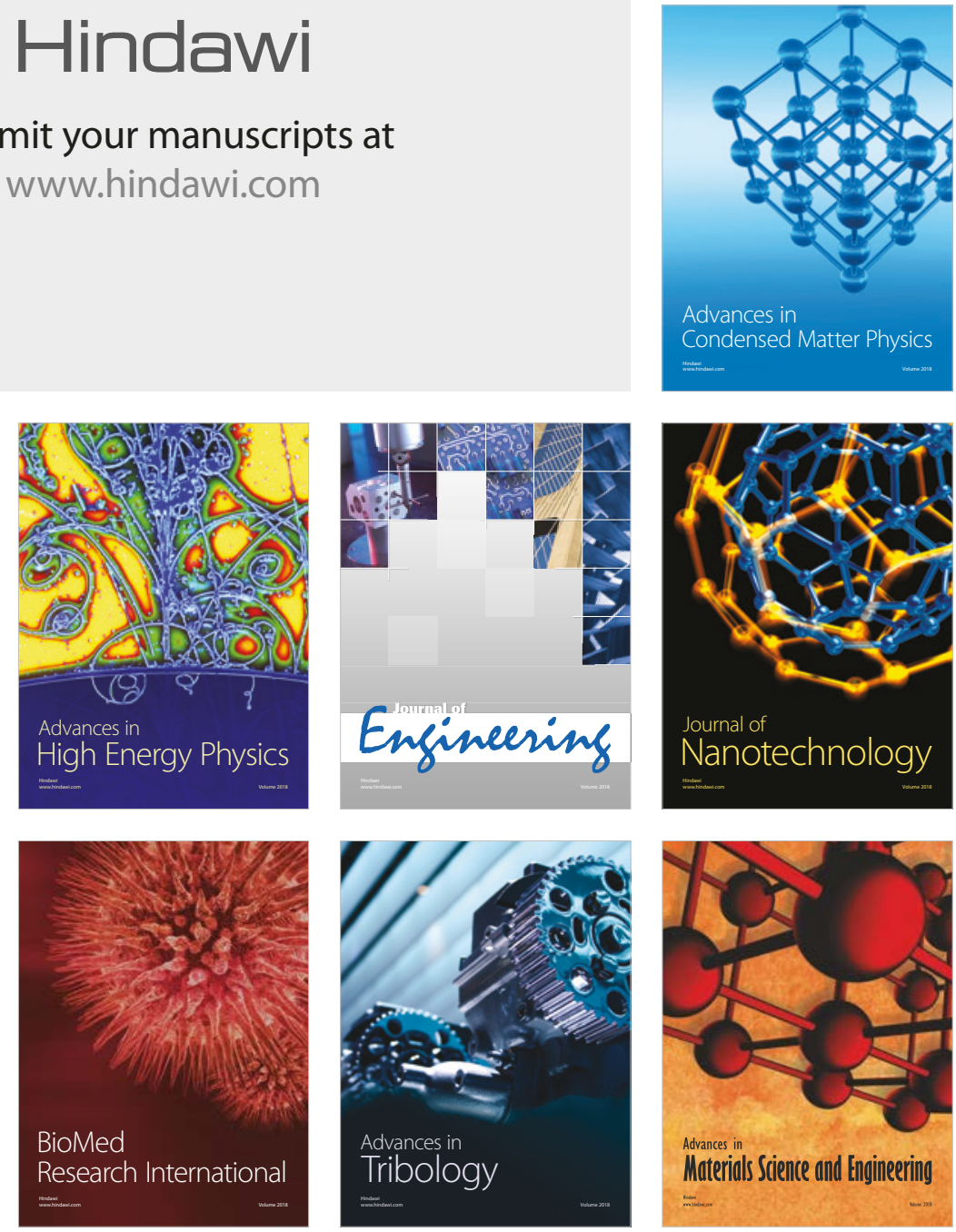